\title{
THE TEMPERATURE STATE OF A PLANE DIELECTRIC LAYER AT CONSTANT VOLTAGE AND FIXED TEMPERATURE OF ONE OF THE SURFACES OF THIS LAYER
}

\author{
Vladimir Zarubin ${ }^{1}$, Georgy Kuvyrkin ${ }^{2}$, and Inga Savelyeva ${ }^{1}$ \\ ${ }^{1}$ Bauman Moscow State Technical University \\ ${ }^{2}$ Bauman Moscow State Technical University National Research University of Technology
}

November 18, 2020

\begin{abstract}
The paper formulates the nonlinear problem of steady-state heat conduction at the constant electric potential difference on the surfaces of a plane dielectric layer with the temperature-dependent heat conduction coefficient and electrical resistivity. A fixed temperature value is set on one of the layer surfaces, and the convective heat exchange with the ambient medium occurs on the opposite surface. The formulation of the problem is transformed into integral ratios, which allows the calculation of the temperature distribution over the layer thickness, governed both by the monotonic and nonmonotonic function. The quantitative assay of the temperature state of a layer of a polymer dielectric made of amorphous polycarbonate is given as an example, as well as the analysis of nonuniformity of the absolute value of electric field intensity over the thickness of this layer.
\end{abstract}

\section{Hosted file}

Text_Zarubin.pdf available at https://authorea.com/users/376956/articles/493772-thetemperature-state-of-a-plane-dielectric-layer-at-constant-voltage-and-fixed-temperatureof-one-of-the-surfaces-of-this-layer 


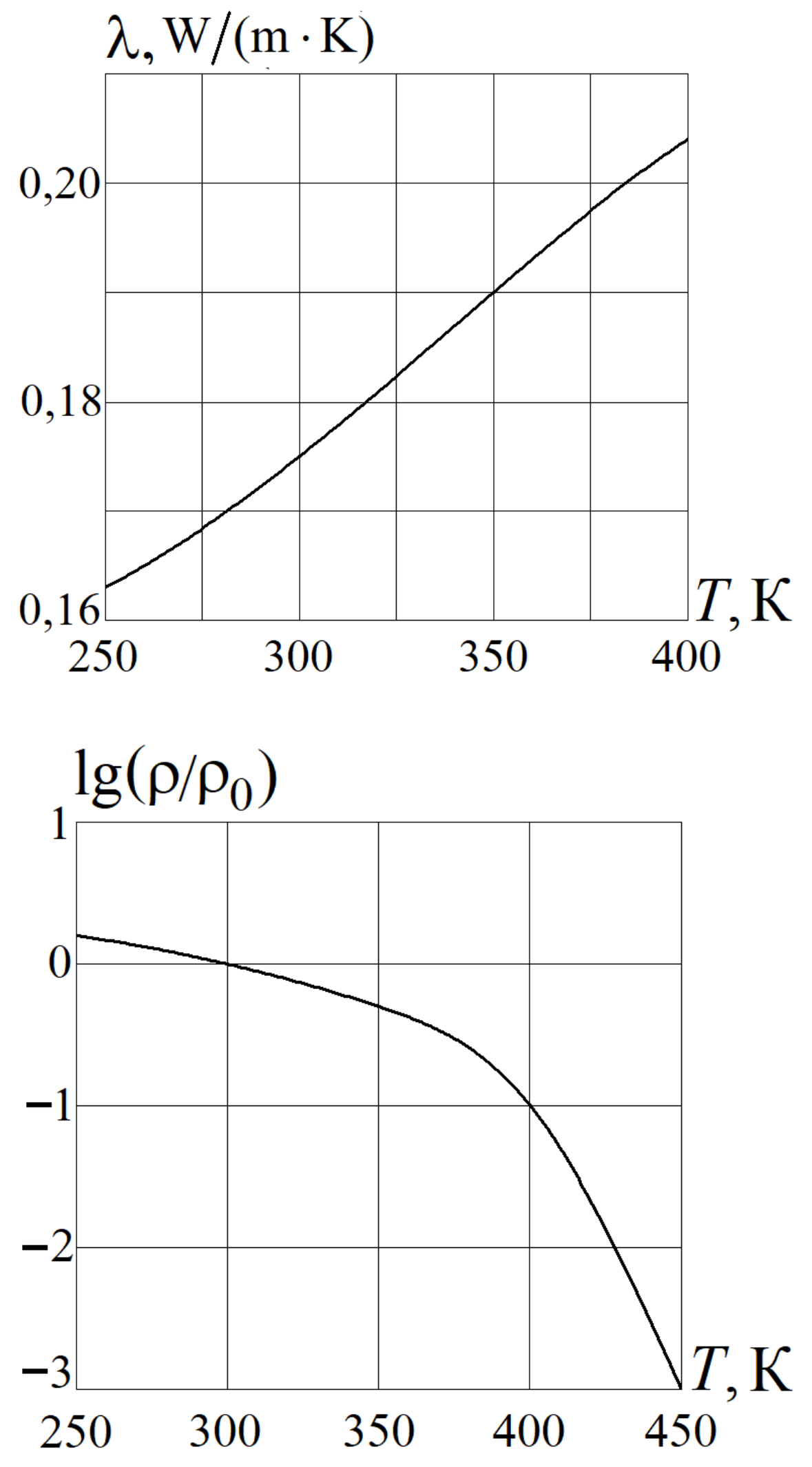



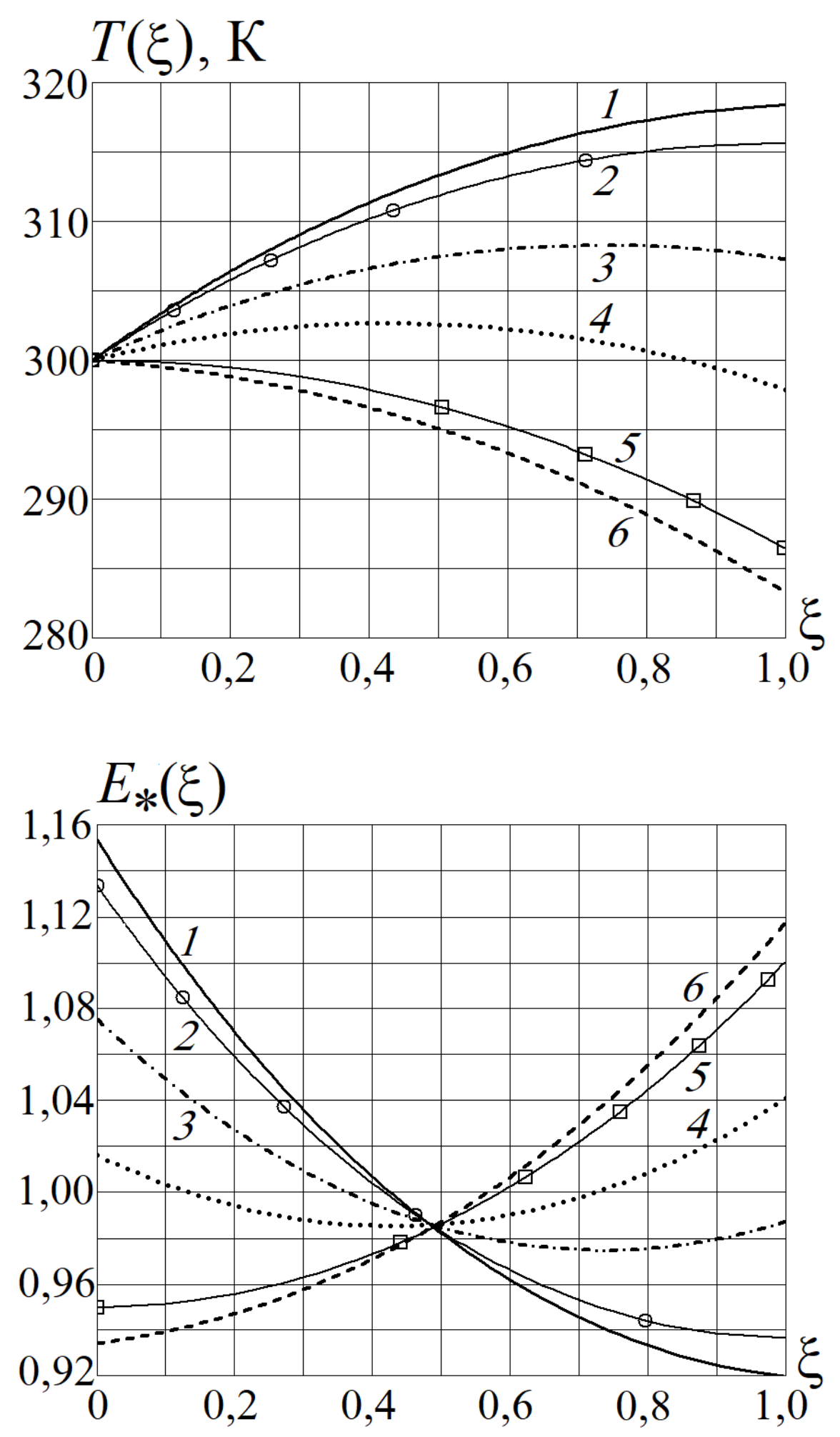